\title{
Manejo endoscópico de osteoma etmoidal con extensión orbitaria: A propósito de un caso
}

\section{Endoscopic manegement of frontoethmoidal osteoma: On case subject}

\author{
David Fuentealba $D^{1}$, Alfredo Naser G1ㄴ Carolina Oliva G², Lucas Ritacco ${ }^{3}$, Gonzalo Miranda $G^{4}$, \\ Rodolfo Nazar S'.
}

\begin{abstract}
RESUMEN
El osteoma es el tumor más frecuente de los senos paranasales, habitualmente asintomático debido a su lento crecimiento, sin embargo, pueden desarrollarse síntomas dependiendo del tamaño, localización y extensión, con potencial compromiso de órbita y cerebro. La cirugía está indicada en casos sintomáticos pudiendo realizarse abordaje externo, endoscópico o combinado. Presentamos un caso de osteoma etmoidal con compromiso orbitario resuelto, manejado por medio de la cirugía endoscópica nasal, con apoyo de navegación.
\end{abstract}

Palabras clave: Osteoma, cirugía endoscópica nasosinusal, frontoetmoidal, navegación, DRAF IIB.

\begin{abstract}
The osteoma is the most common tumor of the paranasal sinuses, is usually asymptomatic because of their slow growth, however, may develop symptoms depending on the size, location and extent, with potential compromise of orbit and brain. Surgery is indicated in symptomatic cases, with external, endoscopic or combined approach. We present a case of ethmoidal osteoma with orbital involvement managed by endoscopic image guided surgery.
\end{abstract}

Key words: Osteoma, endoscopic sinus surgery, frontoethmoidal, navegation, DRAF IIB.

\footnotetext{
Médico Servicio Otorrinolaringología HCUCH.

2 Interna de Medicina, Facultad de Medicina, Universidad de Chile.

3 Médico Unidad de Cirugía Asistida por Computación Hospital Italiano, Buenos Aires.

4 Médico Servicio de Radiología HCUCH.
} 


\section{INTRODUCCIÓN}

El osteoma es el tumor más frecuente de los senos paranasales, correspondiendo a una lesión osteoblástica benigna ${ }^{1}$. Debido a su lento crecimiento habitualmente es asintomático siendo detectado como hallazgo en una tomografía computarizada -TC de cavidades paranasales (CPN)-, sin embargo, pueden desarrollarse síntomas dependiendo del tamaño, localización y extensión de estas lesiones con potencial compromiso de órbita y cerebro. Afecta principalmente el seno frontal, seguido del etmoides, maxilar y esfenoides. En cuanto a su epidemiología los afectados son hombres entre la cuarta y quinta década de la vida, siendo la relación hombre:mujer de 2:12. En casos asintomáticos y en osteomas pequeños el tratamiento es conservador y seguimiento con TC, mientras que en pacientes sintomáticos la remoción quirúrgica es de elección, pudiendo realizarse un abordaje abierto 0 endoscópico ${ }^{3}$. Inicialmente el compromiso de órbita, extensión más allá de la lámina papirácea y gran tamaño del tumor determinaban la vía externa ${ }^{4}$, sin embargo, con el avance de la cirugía endoscópica sinusal se ha reportado éxito en casos complejos, en los cuales el uso de navegación asistida puede aumentar la precisión quirúrgica, ${ }^{5,6}$.
A continuación, se presenta un caso de osteoma etmoidal con compromiso orbitario resuelto gracias a la cirugía endoscópica guiada por imágenes con un sistema de navegación.

\section{CASO CLÍNICO}

Un hombre de 14 años, sin antecedentes mórbidos conocidos, fue evaluado en el Departamento de Rinosinusología del Hospital Clínico de la Universidad de Chile en junio de 2016. Consulta por cuadro de 3 meses de evolución de epífora en ojo izquierdo asociado a diplopía intermitente, aumento de volumen en canto medial de párpado superior izquierdo, de consistencia pétrea, no dolorosa y de lento crecimiento. La evaluación por TC evidenció un extenso osteoma etmoidal izquierdo centrado en la lámina papirácea de diámetros $22 \times 30 \times 28$ mm en sentidos transverso, anteroposterior y longitudinal, respectivamente, el que obstruye el receso nasofrontal y el conducto naso-lagrimal, además de desplazar el músculo recto medial y el globo ocular. En el TC, además se detectó compromiso del seno frontal izquierdo, secundario a la obstrucción del receso del frontal (Figura 1). A la endoscopía nasal

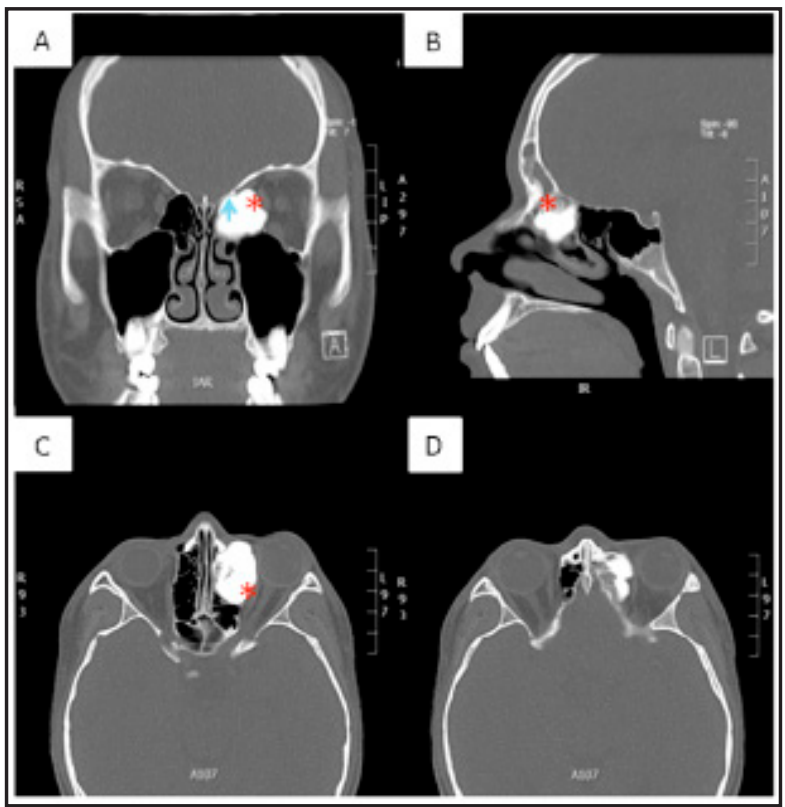

Figura 1. TC de CPN que muestra osteoma etmoidal izquierdo. A) Corte coronal, osteoma desplaza músculo recto medial $\left({ }^{*}\right)$ y contacta con la base de cráneo $(\uparrow)$. B) Corte sagital, se observa el osteoma que obstruye el receso frontal $\left({ }^{*}\right)$, con compromiso inflamatorio secundario del seno. C-D) Corte axial, se observa osteoma que desplaza lámina papirácea, estando en estrecha relación con el músculo recto interno $\left(^{*}\right)$. 
se halló una masa en relación al cornete medio de superficie lisa.

El caso fue discutido en el Departamento de Rinosinusología y se planificó una cirugía endoscópica nasosinusal consistente en una resección endoscópica del osteoma etmoidal asociado a un procedimiento de drenaje del seno frontal del tipo DRAF IIb, asistida por navegación. Se utilizó reconstrucción volumétrica en la planificación operatoria (Figura 2). Al evaluar el TC se evaluaron parámetros favorables a la resección endoscópica del tumor, los cuales fueron: un área de menor densidad del osteoma en relación a la base de cráneo, que aparentemente dejaba un plano de clivaje (Figura 1B), al evaluar la arteria etmoidal anterior, no pasaba de forma libre en la fosa nasal, por lo que el riesgo de lesión de esta estructura durante la cirugía era muy bajo.

Durante el procedimiento se contó con la colaboración del equipo de medicina asistida por computación y navegación del Hospital Italiano de Buenos Aires, que habitualmente trabaja en conjunto con el equipo de rinosinusología para este tipo de casos. Además se contó con el equipo de oftalmología durante la intervención.

Para exponer mejor el osteoma se resecó el hueso lagrimal y la axila del cornete medio preservando el saco y conducto nasolagrimal. Se comenzó fresando desde la parte inferior hacia el centro del osteoma resecando los bordes con pinza Citelli. Al llevar $50 \%$ del volumen fresado, el osteoma se comienza a movilizar, desinsertándose de su base, rodando en $360^{\circ}$ en una especie de "cápsula". Para extraerlo se tracciona hacia la fosa nasal en forma cuidadosa, para evitar fractura de la base del cráneo, se continúa el fresado del osteoma ya libre en la fosa nasal y se fractura en dos partes con pinza Citelli.

Luego de la resección del osteoma, protruyó grasa periorbitaria hacia la fosa nasal sin provocar obstrucción del receso frontal (Figura 3). La biopsia diferida confirma diagnóstico de osteoma. Posteriormente se trasladó al paciente a sala de complejidad estándar, con taponamiento nasal de fosa izquierda, presentando solo edema palpebral leve. Al alta se mantuvo con lavados nasales de alto flujo, corticoides intranasales y antibióticos por 1 semana. En seguimiento periódico evolucionó favorablemente, sin epifora ni diplopia, ni sínto-

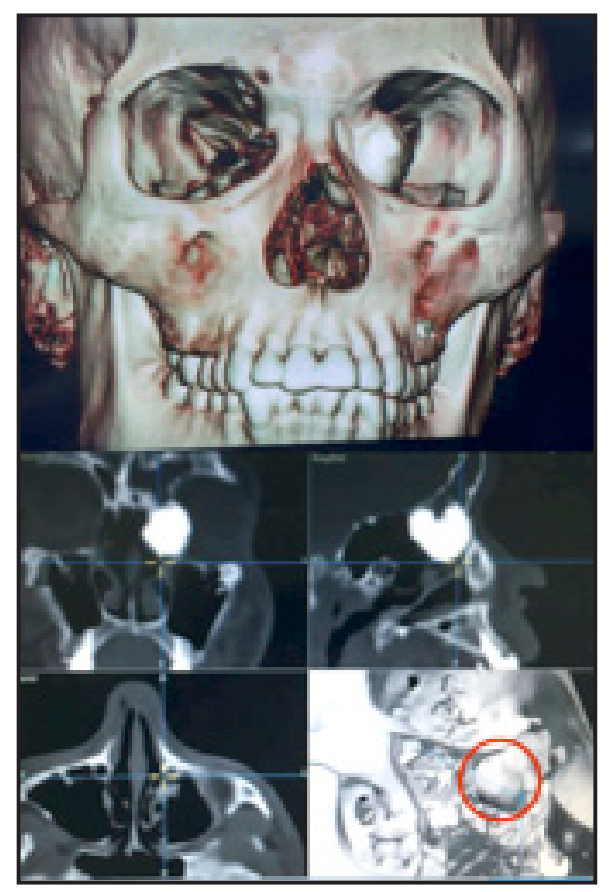

Figura 2. Reconstrucción volumétrica otorgada por sistema de navegación. Se observa la localización del osteoma y sus relaciones anatómicas. Indicado con un círculo se observa la reconstrucción volumétrica tridimensional del tumor. 


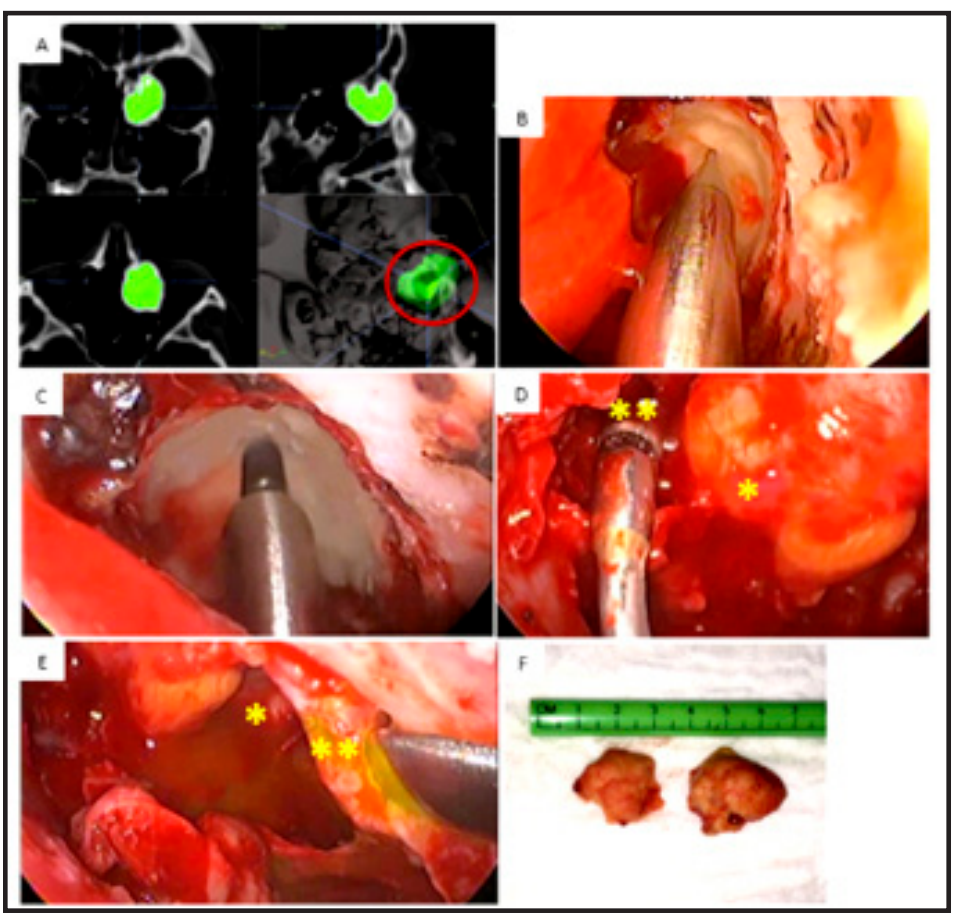

Figura 3. A) Navegación intraoperatoria con imágenes de planeamiento virtual preoperatorio, indicado con un círculo se observa la reconstrucción volumétrica tridimensional del tumor. B) Se observa el puntero de la navegación en el espesor del osteoma para corroborar localización y profundidad del fresado. C) Fresado del osteoma con fresa de alta revolución (30.000 RPM). D) Visión endoscópica de fosa nasal izquierda posresección del osteoma, se observa: grasa orbitaria que protruye a fosa nasal $\left({ }^{*}\right)$, receso del frontal permeable y ampliado $\left(^{* \star}\right)$. E. Visión endoscópica de fosa nasal izquierda posresección de osteoma, se observa: seno maxilar $\left({ }^{*}\right)$, conducto lagrimal permeable, al irrigar fluoresceína. $\left(^{* \star}\right)$. F. Osteoma resecado en su totalidad luego del fresado.

mas nasales, puede observarse en la Figura 4 los resultados posoperatorios en TC tomado 5 meses luego de la intervención quirúrgica, que demuestra indemnidad de la base de cráneo, receso frontal permeable y protrusión de grasa periorbitaria hacia la fosa nasal, sin complicaciones.

\section{DISCUSIÓN}

El osteoma es el tumor más frecuente de los senos paranasales. Solo el $4 \%-10 \%$ de todos los osteomas producen síntomas, relacionados a su tamaño, localización y tasa de crecimiento. El diagnóstico generalmente es accidental a través del TC de CPN, que es la imagen de elección ya que permite determinar el tamaño, localización, extensión y enfermedad nasosinusal concomitante.
Aproximadamente el $3 \%-6,4 \%$ de TC de CPN revelan osteomas ${ }^{2}$. Su patrón corresponde a una lesión radiopaca bien definida con un centro radiolúcido rodeada por esclerosis densa. En ocasiones la RNM es útil en el diagnóstico diferencial, en confirmar la asociación a mucocele y en casos de compromiso intraorbitario?.

El manejo conservador se prefiere para osteomas pequeños 0 asintomáticos, a través de seguimiento con TC cada 1 a 2 años, esto debido a que la tasa de crecimiento promedio es de $1,61 \mathrm{~mm}$ al año, con un rango 0,44-6,0 $\mathrm{mm}^{8}$.

El manejo quirúrgico se utiliza en casos sintomáticos y depende de su ubicación, pudiendo realizarse un abordaje endoscópico, externo o combinado, siendo su objetivo la remoción total del tumor en forma segura sin lesión de órganos adyacentes 9 . Las indicaciones quirúrgicas para osteomas fronto- 


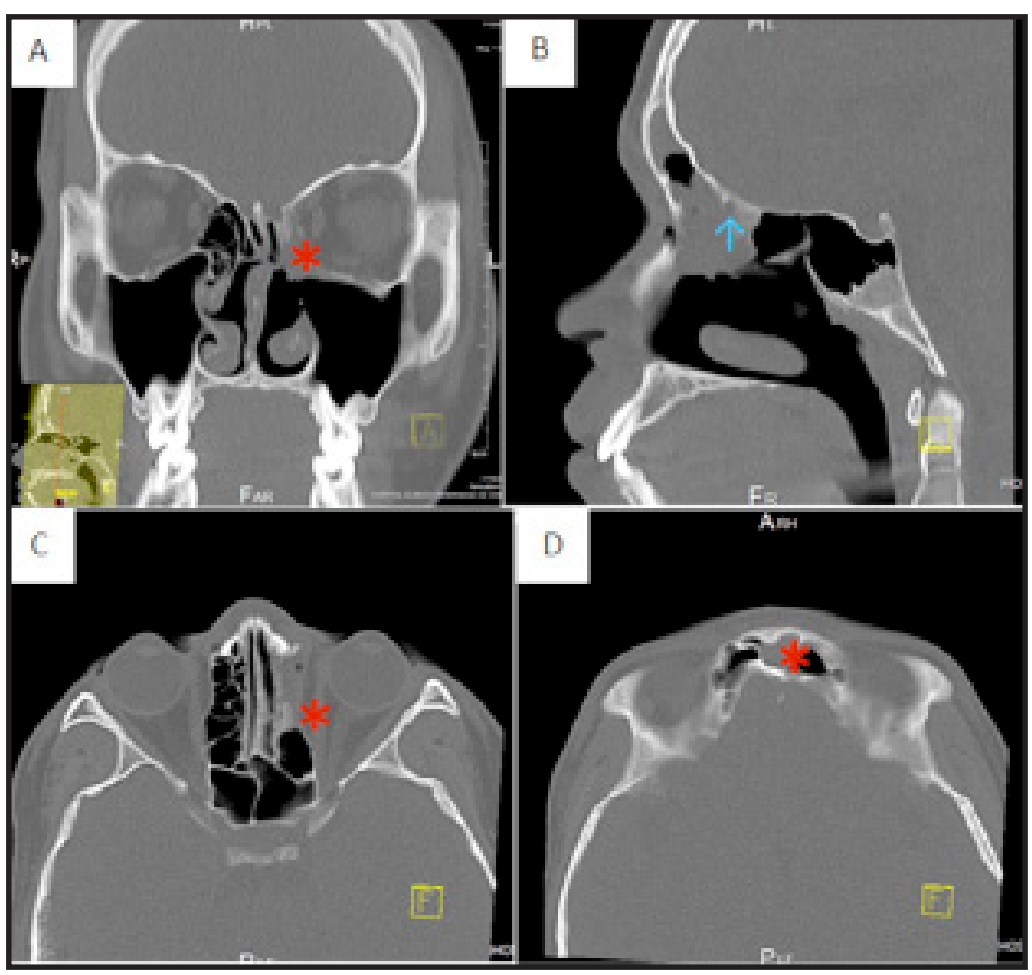

Figura 4. TC de control posoperatorio. A) Corte coronal. Se observa desplazamiento hacia fosa nasal de grasa periorbitaria $\left(^{*}\right)$. B) Corte sagital se observa indemnidad de la base de cráneo $(\uparrow)$. C) Corte axial. Se evidencia músculo recto interno en posición anatómica $\left({ }^{*}\right)$. D) Corte axial, se observa receso del frontal permeable, con cambios inflamatorios secundario a cuadro agudo $\left({ }^{*}\right)$.

etmoidales son: aquellos que sobrepasa los límites del seno frontal, compromiso mayor al $50 \%$ del volumen del seno, crecimiento continuo, que obstruya el receso del frontal (como el presente caso), cefalea sin otra causa que la explique, asociados a mucocele, síntomas orbitarios y neurológicos ${ }^{7,10}$.

El abordaje externo está indicado cuando existen tumores grandes (mayor a $3 \mathrm{~cm}$ ), tumores que comprometen ambos senos frontales 0 estructuras nobles y aquellos que crecen en la tabla interna del seno². El flap osteoplástico (FOP) con obliteración grasa, fue considerado por mucho tiempo la mejor técnica para el manejo del seno frontal, otros abordajes externos son la rinotomía lateral, cirugía de Caldwell-Luc y frontoetmoidectomía externa ${ }^{1,7,11}$. Las ventajas de esta vía son proveer una excelente visualización del seno incluyendo pared superior, posterior y lateral (aunque no siempre del receso nasofrontal y etmoides), instrumentación bimanual, mejor control de potenciales complicaciones intraoperatorias como sangrado y fístula de LCR, además de ser una técnica ampliamente disponible ${ }^{12}$.

Clásicamente el abordaje endoscópico se ha utilizado en tumores pequeños 0 restringidos a un solo seno, siendo ideal en tumores confinados al seno etmoidal y cavidad nasal. El principal objetivo es reducir el volumen por medio de una técnica de cavitación con fresado endoscópico (desde la periferia al centro), fracturar la lesión y remover los fragmentos ${ }^{13}$. Existen dificultades en este abordaje debido a que la anatomía es estrecha por la dimensión del receso frontal y del ostium, y acceso limitado al receso supraorbitario; a lo que se suma la mala visualización y el pobre acceso de la parte lateral del seno, particularmente en el piso. Para esta área se accede mejor por la cavidad 
nasal contralateral a través de una perforación en el septo superior ${ }^{14}$.

En el año 2001 Schick y Draf reportaron la primera serie con técnica microscópica transnasal para remover osteoma del frontal ${ }^{15}$. Chiu y cols recomendaron el abordaje endoscópico para resección de osteomas pequeños frontales ubicados medial a una línea sagital que pasa a través de la lámina papirácea; y utilizando la vía externa si la localización es lateral a ésta o si el seno frontal está completamente comprometido ${ }^{16}$. Se han reportado contraindicaciones a la cirugía endoscópica exclusiva como diámetro anteroposterior del seno frontal menor a $1 \mathrm{~cm}$, localización superior extensa con más de $2 \mathrm{~cm}$ de osteoma en el seno frontal y localización lateral más allá de la pared medial de la órbita ${ }^{3}$. Sin embargo, Turri Zanoni sugirió que el tamaño de la lesión, extensión lateral que sobrepasa la lámina papirácea y compromiso intraorbitario no extenso, no son contraindicaciones absolutas para resección endoscópica pura ${ }^{4}$, por lo que la decisión en estas situaciones debe ser tomada basada en la experiencia del cirujano y caso a caso. En nuestro caso existía compromiso lateral a la lámina papirácea, lo cual no fue contraindicación para cirugía endoscópica.

Para osteomas etmoidales puros existe menos referencia en la literatura en su manejo. El compromiso de la lámina cribosa no es una contraindicación e incluso con compromiso orbitario puede ser abordado por vía endoscópica gracias a los avances en cirugía endoscópica sinusal ${ }^{17,18}$. Los osteomas pequeños pueden removerse por medio de resección en bloque, si el osteoma es excepcionalmente largo y ampliamente unido a los bordes etmoidales, el tumor puede reducirse en tamaño con el uso de fresa antes de su remoción completa$^{2}$. La extensión anterior al conducto lagrimal y por debajo de la piel pueden requerir un abordaje endoscópico combinado ${ }^{7}$.

En cuanto a las ventajas de la vía endoscópica, ésta permite un tiempo hospitalario más corto, preserva el drenaje natural endonasal, disminuye la morbilidad posoperatoria, posee mejores resultados cosméticos, además de la utilización de cirugía guiada por imágenes como en este caso. Entre sus desventajas destacan un tiempo operatorio mayor y complicaciones como fístula de LCR o injuria orbitaria endoscópica3,19.
Es necesario una planificación preoperatoria con el fin de evaluar la dificultad técnica de un abordaje endoscópico $\mathrm{v} / \mathrm{s}$ abierto ${ }^{20}$. En nuestro caso las características anatómicas críticas fueron: seno frontal con diámetro anteroposterior adecuado, gran extensión de la lesión hacia etmoides y órbita (con íntima relación con tróclea del músculo oblicuo superior), zona de implantación en base de cráneo anterior en un área de menor densidad y posterior al receso del frontal. El tumor se encontraba en íntima relación con la arteria etmoidal anterior. Además de las dificultades anatómicas debe tomarse en consideración la experiencia del equipo quirúrgico para decidir un abordaje endoscópico. Inicialmente expusimos completamente el osteoma, para lo cual fue necesario remover la cabeza del cornete medio y el hueso lagrimal con precaución de no lesionar el conducto nasolagrimal. Para no lesionar la órbita se comenzó a fresar hacia el interior de osteoma de manera de adelgazar sus paredes. En el intraoperatorio el osteoma se desinsertó de la base de cráneo quedando contenido en una especie de cápsula, para evitar lesiones decidimos fracturar en dos el osteoma y bajarlo de la base de cráneo a la fosa nasal para su posterior retiro. Al revisar posteriormente el lecho del osteoma, la grasa periorbitaria se hernió hacia la fosa nasal lo cual no presentó mayor complicación, y quedó la base de cráneo intacta y receso del frontal amplio.

Recomendamos que para la resección endoscópica de un osteoma grande, se considere una cirugía endoscópica funcional con abordaje extendido DRAF III o IIB, asistencia por navegación, fresado con motor M5 (30.000 RPM), técnica de cavitación con fresado endoscópico ("dejar el osteoma con una tela de cebolla") ${ }^{13}$, identificación del sitio de inserción y manejo en conjunto con equipo de oculoplástica.

La cirugía guiada por imágenes con sistema de navegación y reconstrucción volumétrica es claramente útil en la cirugía endoscópica de osteoma, ofreciendo mayor exactitud, remoción completa del osteoma y preservación de las estructuras vitales que lo rodean como la órbita o cerebro, siendo reportada anteriormente con satisfactorios resultados ${ }^{5,6}$. En nuestro caso permitió una buena delimitación de base de cráneo y órbita, precisar profundidad del fresado y resección completa del 
tumor. Sin embargo, a pesar de otorgar mayor exactitud no se ha demostrado una disminución en las complicaciones en comparación a la cirugía endoscópica funcional sin navegación, probablemente debido a que la CEF reporta muy bajas complicaciones $(3 \%)$ requiriendo un número de pacientes demasiado alto para demostrar diferencias significativas ${ }^{21,22}$.

\section{CONCLUSIÓN}

Los osteomas de senos paranasales son lesiones benignas usualmente asintomáticas, aunque algunos pacientes son sintomáticos siendo necesaria una imagen TC para su evaluación.
El manejo quirúrgico está indicado en tumores sintomáticos y rápidamente progresivos, las opciones incluyen abordaje externo, endoscópico o su combinación. La decisión debe ser tomada basada en la localización y tamaño. Aunque se ha recomendado la vía externa para casos complejos por dificultades anatómicas, compromiso de órbita y base de cráneo, se ha incrementado el abordaje endoscópico debido a sus avances, entre los cuales se incluyen la implementación de cirugía asistida por imágenes mediante navegación, otorgando mayor seguridad y precisión anatómica en tiempo real.

A nuestro entender el abordaje endoscópico nasosinusal, en este tipo de tumores debiera ser el de elección, siempre que se cuente con el equipamiento y entrenamientos adecuados.

\section{BIBLIOGRAFÍA}

1. Strek P, Zagólski 0, SkŁadzien J, Kurzynski $M$, Drduch G. Osteomas of the paranasal sinuses:surgical treatment options. Med Sci Monit 2007; 13(5): CR244-50.

2. Muderris T, Bercin S, Sevil E, Kiris M. Endoscopic removal of a giant ethmoid osteoma with orbital extension. Acta Inform Med 2012; 20(4): 266-8.

3. Lee DH, Jung SH, Yoon TM, Lee JK, Joo YE, Lim SC. Characteristics of paranasal sinus osteoma and treatment outcomes. Acta Otolaryngol 2015; 135(6): 602-7.

4. Turri-Zanoni M, Dallan I, Terranova P, Battaglia P, Karkigkiotis A, Bignami M, Castelnuovo P. Frontoethmoidal and intraorbital osteomas: exploring the limits of the endoscopic approach. Arch Otolaryngol Head Neck Surg 2012; 138(5): 498-504.

5. Wang Y, Li D, Jiang A, Zhang H, Sun Y, Liu Z, Song X. Endoscopic Resection of Giant Ethmoid Osteomas with the Assistance of an Image-guidance System. Anticancer Res 2016; 36(3): 1325-30.

6. Song $X C$, Chen LY, Zhang QQ, Sun $Y$, Wang $Q$, Zhang H, Chen XM, Wang Y. Endoscopic removal of ethmoid osteomas under navigation guidance. Zhonghua Er Bi Yan Hou Tou Jing Wai Ke Za Zhi 2011; 46(2): 91-5.

7. Georgalas C, Goudakos J, Fokkens WJ. Osteoma of the skull base and sinuses. Otolaryngol Clin North Am 2011; 44(4): 875-90, vii.

8. Koivunen $P$, Löppönen $H$, Fors AP, Jokinen $K$. The growth rate of osteomas of the paranasal sinuses. Clin Otolaryngol Allied Sci 1997; 22(2): 111-4.

9. Celenk F, Baysal E, Karata ZA, Durucu C, Mumbuc S, Kanlikama M. Paranasal sinus osteomas. J Craniofac Surg 2012; 23(5): e433-7.

10. Savic DL, DJeRIC DR. Indications for the surgical treatment of osteomas of the frontal and ethmoid sinuses. Clin Otolaryngol Allied Sci 1990; 15(5): 397-404.

11. Rokade A, Sama A. Update on management of frontal sinus osteomas. Curr Opin Otolaryngol Head Neck Surg 2012; 20(1): 40-4.

12. Castelnuovo P, Valentini V, Giovannetti F, Bignami M, Cassoni A, Iannetti G. Osteomas of the maxillofacial district: endoscopic surgery versus open surgery. J Craniofac Surg 2008; 19(6): 1446-52.

13. Turri-Zanoni M, Dallan I, Terranova P, Battaglia P, Karligkiotis A, Bignami M, Castelnuovo P. Frontoethmoidal and intraorbital osteomas: exploring the limits of the endoscopic approach. Arch Otolaryngol Head Neck Surg 2012; 138(5): 498-504. doi: 10.1001/archoto.2012.644. 
14. Gotlib T, Held-Ziokowska M, Niemczyk K. Frontal sinus and recess osteomas: an endonasal endoscopic approach. B-ENT2014; 10(2): 141-7.

15. Schick B, Steigerwald C, el Rahman el Tahan $A$, Draf W. The role of endonasal surgery in the management of frontoethmoidal osteomas. Rhinology 2001; 39(2): 66-70.

16. Chiu AG, Schipor I, Cohen NA, Kennedy DW, Palmer JN. Surgical decisions in the management of frontal sinus osteomas. Am J Rhinol 2005; 19(2): 191-7.

17. Miman MC, Bayindir T, Akarcay M, Erdem T, Selimoglu E. Endoscopic removal technique of a huge ethmoido-orbital osteoma. J Craniofac Surg 2009; 20(5): 1403-6.

18. Torun MT, Turan F, Tuncel Ü. Giant ethmoid osteoma originated from the lamina papyracea.
Med Arch 2014; 68(3): 209-11.

19. Castelnuovo P, Giovannettı F, Bignamı M, Ungari $C$, Iannetti $G$. Open surgery versus endoscopic surgery in benign neoplasm involving the frontalsinus. J Craniofac Surg 2009; 20(1): 180-3.

20. Ximena Maul F, Claudia González G, Claudio Callejas C. Osteoma frontoetmoidal, otras aplicaciones del Draf III, a raíz de un caso clínico. Rev Otorrinolaringol Cir Cabeza Cuello 2015; 75: 245-50.

21. Ramakrishnan VR, Kingdom TT. Does Image-Guided Surgery reduce complications? Otolaryngol Clin North Am 2015; 48(5): 851-9.

22. Smith TL, Stewart MG, Orlandi RR, Setzen M, LANZA DC. Indications for image-guided sinus surgery: the current evidence. Am J Rhino/2007; 21: $80-3$.

Dirección: Rodolfo Nazar S

Servicio de ORL Hospital Clínico de la Universidad de Chile

E mail: rnazars@gmail.com 